\title{
A Segmentation Model Using Compound Markov Random Fields Based on a Boundary Model
}

\author{
Jue Wu, Student Member, IEEE, and Albert C. S. Chung
}

\begin{abstract}
Markov random field (MRF) theory has been widely applied to the challenging problem of image segmentation. In this paper, we propose a new nontexture segmentation model using compound MRFs, in which the original label MRF is coupled with a new boundary MRF to help improve the segmentation performance. The boundary model is relatively general and does not need prior training on boundary patterns. Unlike some existing related work, the proposed method offers a more compact interaction between label and boundary MRFs. Furthermore, our boundary model systematically takes into account all the possible scenarios of a single edge existing in a $3 \times 3$ neighborhood and, thus, incorporates sophisticated prior information about the relation between label and boundary. It is experimentally shown that the proposed model can segment objects with complex boundaries and at the same time is able to work under noise corruption. The new method has been applied to medical image segmentation. Experiments on synthetic images and real clinical datasets show that the proposed model is able to produce more accurate segmentation results and satisfactorily keep the delicate boundary. It is also less sensitive to noise in both high and low signal-to-noise ratio regions than some of the existing models in common use.
\end{abstract}

Index Terms-Boundary model, Markov random fields (MRFs), medical image segmentation.

\section{INTRODUCTION}

I MAGE segmentation is a task that classifies pixels of an image using different labels so that the image is partitioned into nonoverlapping labeled regions. Extraction of regions or objects of interest is usually the first important step in almost every task of image processing and high-level image analysis and understanding. Although it is basic, image segmentation is one of the most difficult problems that researchers are facing because most of the real objects have complex shapes, boundaries and morphology, and true images are often corrupted by noise that cannot be ignored.

To tackle the difficult problem of image segmentation, researchers have proposed a variety of methods. Segmentation using the Markov random field (MRF) modeling is characterized by probability distributions of site-interacting properties and neighboring restriction. The most notable property of the MRF model is that the conditional probability of one site over

Manuscript received September 6, 2005; revised May 16, 2006. This work was supported by the Research Grants Council (RGC) of Hong Kong under Grant HKUST6209/02E. The associate editor coordinating the review of this manuscript and approving it for publication was Dr. Giovanni Poggi.

The authors are with the Lo Kwee-Seong Medical Image Analysis Laboratory, Department of Computer Science and Engineering, and Bioengineering Program, School of Engineering, The Hong Kong University of Science and Technology, Hong Kong (e-mail: johnwoo@ust.hk; achung@cs.ust.hk).

Digital Object Identifier 10.1109/TIP.2006.884933 all the others is only dependent on the relation of the site over its neighbors. This property, called Markovianity, embodies the spatial interactions of adjacent sites and offers a way to incorporate prior information into the MRF models. The advantages of MRF modeling are as follows. It has a relatively simple and effective architecture for embedding the prior and likelihood probabilities. It also takes into consideration the contextual constraints while maintaining the complexity at a tractable level by keeping the size of the neighborhood system relatively small. In terms of mathematical functional optimization, i.e., maximum a posteriori (MAP), MRF models make many complex problems (including segmentation) in image processing computationally feasible and can give reasonable results.

When the MRF theory was first introduced into the field of statistical image analysis in the mid-1980s, Geman and Geman [1] and Besag [2] applied MRFs to image restoration, which can be viewed as a generalization of segmentation. Similar to the work of Geman and Geman [1], Geiger and Girosi [3] also added a second MRF (line process) to the original MRF for surface reconstruction. Likewise, in the work of Jeng and Woods [4] and Molina et al. [5], line process (edge MRF) was incorporated into the intensity process (label MRF). In general, adopting two or more MRFs in one task is a way to solve two or more different problems. For example, Sun et al. [6] integrated three MRFs, disparity, line process and occlusion, for stereo problems because these three factors are all critical to stereo matching. Similarly, Arduini et al. [7] solved two problems, restoration of SAR images and extraction of intensity discontinuities, by using two distinct MRFs. Held et al. [8] used one added MRF, i.e., the bias field, to sweep the obstacle of MRI brain segmentation but they did not couple the two MRFs compactly because the two fields are assumed independent.

In this paper, rather than using a single label MRF, we incorporate a new MRF (boundary MRF) to represent the boundary of a region and, thus, construct a compound MRF model like [1], [3]-[7]. Our boundary MRF is different from the line process as we define the MRF not on the dual lattice between pixels but on the pixel site directly. Moreover, in our model, the two MRFs (label and boundary MRFs) interact in a more sophisticated way while the line process works implicitly [9] and is relatively simple. Rather than tackling two or more aspects of a problem simultaneously [1], [3]-[7], our current model aims at image segmentation alone, with the incorporation of a boundary MRF in order to increase the segmentation accuracy. We consider and construct more complex interactions of the two MRFs (label and boundary) in a neighborhood by preferring a series of label patterns and penalizing the other situations with a basic assumption that true boundaries should be reasonably linked and 
matched with these label patterns while discontinuities caused by random noise are not. In the experiments, we use a number of simulated images with different signal-to-noise ratios (SNR) and some real brain MRI datasets to test our method and other existing MRF models. The results show that the new model can give higher segmentation accuracy while preserving the extracted object boundary well. Other work applying MRF models to brain image segmentation can be found in [8], [10]-[14].

The theme of this paper is basically the same as our previous conference paper [15], but we modify the boundary model and make it more systematic and less heuristic. More explanation of the ideas and formulation can be found in the following sections. In Section II, the proposed model is introduced in detail. Section III presents the control models and the experimental results of comparison. Then, some discussions are made in Section IV. We conclude this paper with Section V.

\section{MODEL FORMULATION}

In this section, we introduce our coupled MRF model that is formulated in a probabilistic framework based on the Bayesian theory. The elements of the framework are given and details on the boundary model and the coupling of two MRFs (label and boundary MRFs) are discussed.

\section{A. MAP-MRF Framework}

Let $S=\{1, \ldots, n\}$ index $n$ sites in an image lattice, supposing that the image of interest has $n$ pixels. $X=\left\{x_{i} \mid i \in S\right\}$ and $D=\left\{d_{i} \mid i \in S\right\}$ are two MRFs representing label tag and boundary tag, respectively. $x_{i}$ is assigned one of the labels in $L_{1}=\{0,1, \ldots, m-1\}$ where $m$ represents the number of possible classes. $d_{i}$ belongs to one of the binary tags in $L_{2}=\{0,1\}$, where 0 and 1 represent nonboundary and boundary sites, respectively. The observed field is denoted by $Y=\left\{y_{i} \mid i \in S\right\}$, where $y_{i}$ is the known image intensity. Let $\Omega_{X}=L_{1} \times \cdots \times$ $L_{1}=L_{1}^{n}$ and $\Omega_{D}=L_{2} \times \cdots \times L_{2}=L_{2}^{n}$ be the configuration spaces of the label MRF $X$ and boundary MRF $D$, respectively.

Advocated by Geman and Geman [1] and others, the maximum a posteriori (MAP) approach is commonly used to estimate the optimal solution of MRF models. This MAP-MRF framework allows us to develop algorithms systematically based on the Bayesian decision and estimation theory. The posterior probability $P(X, D \mid Y)$ in our model represents the joint probability of label and boundary MRFs, $X$ and $D$, given the observed intensity field $Y$ and can be estimated using the Bayes' theorem

$$
P(X, D \mid Y)=\frac{P(Y \mid X, D) P(X, D)}{P(Y)}
$$

where $P(Y \mid X, D)$ reflects the likelihood of the observed intensity values given the information of labels and boundaries in an image; $P(X, D)$ embodies the joint prior knowledge of the label MRF $X$ and boundary MRF $D$; and $P(Y)$ is the likelihood of the observed intensity values. Since the observed intensity values are known and unchanged, $P(Y)$ is thought to be constant so that (1) further leads to
$P(X, D \mid Y) \propto P(Y \mid X, D) P(X, D)$. The MAP estimation for the optimal solution is then estimated by

$$
(\hat{X}, \hat{D})=\arg \max _{X \in \Omega_{X}, D \in \Omega_{D}} P(Y \mid X, D) P(X, D)
$$

where $\hat{X}$ is the final segmented image that we target.

By virtue of the Markovianity of MRF theory, interactions between sites in $S$ are constrained in a neighborhood system $\mathcal{N}=\left\{N_{i} \mid i \in S\right\}$, where $N_{i} \subseteq S$ denotes a set of sites in the vicinity of site $i$. According to the Hammersley-Clifford theorem, $X$ is an MRF with respect to $\mathcal{N}$ if and only if $P(X)$ is a Gibbs distribution with respect to $\mathcal{N}$. A Gibbs distribution of $X$ is given by

$$
P(X)=\frac{1}{Z} e^{-U(X) / T}
$$

where $T$ is a temperature constant, $U(X)$ is an energy function and $Z$ is a normalizing constant. Supposing that the likelihood function can be expressed in Gibbs distribution, the MAP estimation becomes

$$
(\hat{X}, \hat{D})=\arg \max _{X \in \Omega_{X}, D \in \Omega_{D}} \frac{1}{Z} e^{-(U(Y \mid X, D)+U(X, D)) / T}
$$

where $U(Y \mid X, D)$ and $U(X, D)$ are the likelihood and prior energy functions, respectively. This further leads to an energy minimization problem, i.e.,

$$
(\hat{X}, \hat{D})=\arg \min _{X \in \Omega_{X}, D \in \Omega_{D}}(U(Y \mid X, D)+U(X, D))
$$

where $\hat{X}$ is the solution to the segmentation problem. We assume that the intensity field $Y$ and the boundary MRF $D$ are independent of each other because the observed image intensity is not affected whether the site is on the region boundary or inside the region. Therefore, the likelihood energy becomes

$$
U(Y \mid X, D)=U(Y \mid X)
$$

Assuming that each region is without texture and nearly homogeneous before it is corrupted by a Gaussian noise with zero mean and standard deviation $\sigma$, we can formulate the likelihood energy as

$$
U(Y \mid X)=\sum_{i \in S} \frac{\left(y_{i}-\mu_{x_{i}}\right)^{2}}{2 \sigma^{2}}
$$

where $\mu_{j}$ represents the mean intensity of region $j\left(j \in L_{1}\right)$. The standard deviation (SD) $\sigma$ can also be dependent on region class. In that case, only a small change is needed to make in (7), and we should use $m$ different SDs, $\sigma_{j}, j=0, \ldots, m-1$ for each region $j$. However, in this paper, since we assume that an image is corrupted by independent and identically distributed (i.i.d.) noise, we use a uniform noise SD for the whole image. The formulation of the prior energy function is introduced in the next subsection. 


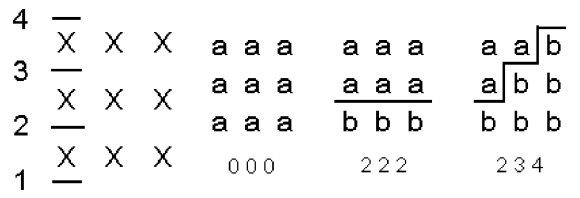

$\begin{array}{lll}\text { (a) } & \text { (b) } & \text { (c) }\end{array}$

Fig. 1. Subfigure (a) illustrates the numbering of edge positions along a column according to its height (4 and 1 represent top and bottom edges, respectively, and 0 represents no edge in the column). The rest [(b)-(d)] are examples of some edges passing through a $3 \times 3$ window and their corresponding numbers. For example, (b) shows no edges in three columns of the $3 \times 3$ windrow and, thus, gives the number: 000. The letters "a" and "b" represent two distinct region labels.

\section{B. Coupling Label MRF With Boundary MRF}

The prior energy $U(X, D)$ defines the interactions between the label MRF $X$ and boundary MRF $D$ and is the major contribution of this paper. Here, we adopt a general model that does not need prior training about the boundary patterns. We assume that a boundary is part of a region and an edge is located between two regions (boundaries). If all the first-order neighbors of a pixel and the pixel itself belong to the same region, we regard this pixel as inside one region and not a boundary point. Otherwise, it is located on the boundary of its region. An edge belongs to no region and actually is on the dual lattice of the image. We systematically study the situations when a single edge passes through a $3 \times 3$ window to see what the likely configurations of the two MRFs, $X$ and $D$ are. On the assumption that the boundary of the object of interest is linked and continuous, we select a number of preferable cases from all the possible combinations of $X$ and $D$ configurations in $\mathcal{N}$ and penalize the other cases. In the energy minimization framework, as stated in (5), preferred cases should make the energy $U(X, D)$ low and the penalized ones should make the energy value high. For instance, the case where site $i$ and all its first-order neighbors $j \in N_{i}$ are labeled as the same class and there is no boundary site in $N_{i} \cup\{i\}$ is preferable so we assign a low energy value to it. The case can be formulated as an energy function by

$$
\gamma \cdot \delta\left(d_{i}\right)\left(\sum_{j \in N_{i}} x_{i} \oplus x_{j}+\sum_{j \in N_{i}} d_{j}\right), \gamma>0
$$

where if $x=0, \delta(x)=1$; else $\delta(x)=0$. The sign $\oplus$ represents the "exclusive or" operation and $\gamma$ is the penalty if the configuration does not belong to this case. The configuration of label MRF $X$ is illustrated in Fig. 1(b), and (8) corresponds to row 1 in Table II (more explanations about the configurations and table are given later in this section). For another example, we also prefer the case where there is a straight edge passing through the $3 \times 3$ window as illustrated in Fig. 1(c). This case can be formulated by

$$
\gamma \cdot \delta\left(d_{i}-1\right)\left(\left|\sum_{j \in N_{i}} x_{i} \oplus x_{j}-1\right|+\left|\sum_{j \in N_{i}} d_{j}-3\right|\right), \gamma>0 .
$$

TABLE I

EACH Row Lists the CONFIGURATION CASES THAT ARE EQUivalENT AFTER FLIPPING OR ROTATION. EVERY ROW CORRESPONDS To ONE CASE IN Fig. 2, IN Which There are 20 CASES

\begin{tabular}{|l|}
\hline Isomorphic configuration codes \\
\hline 111,444 \\
\hline $112,211,344,443$ \\
\hline $113,311,244,442$ \\
\hline 121,434 \\
\hline $122,221,334,433$ \\
\hline $213,312,243,342$ \\
\hline 222,333 \\
\hline $223,233,322,332$ \\
\hline 232,323 \\
\hline 313,242 \\
\hline $123,321,234,432$ \\
\hline 131,424 \\
\hline $132,231,324,423$ \\
\hline $133,331,224,422$ \\
\hline 212,343 \\
\hline $114,144,411,441$ \\
\hline 141,414 \\
\hline $142,413,314,241$ \\
\hline $143,412,214,341$ \\
\hline $124,421,431,134$ \\
\hline
\end{tabular}

This formulation corresponds to row 9 of Table II. If we consider the two above instances simultaneously, the energy function should be given as

$$
\begin{aligned}
& \sum_{i \in S} \gamma \cdot\left[\delta\left(d_{i}\right) t_{1}(i)+\delta\left(d_{i}-1\right) t_{2}(i)\right], \gamma>0 \\
& t_{1}(i)=\sum_{j \in N_{i}} x_{i} \oplus x_{j}+\sum_{j \in N_{i}} d_{j} \\
& t_{2}(i)=\left|\sum_{j \in N_{i}} x_{i} \oplus x_{j}-1\right|+\left|\sum_{j \in N_{i}} d_{j}-3\right| .
\end{aligned}
$$

Equation (10) is actually a combination of (8) and (9).

We explore all the possible scenarios of a single edge passing through a $3 \times 3$ window. They are listed in Fig. 2 . In the figure, we list up to 73 pairs of MRF $X$ and $D$ configurations in a $3 \times 3$ window, e.g., 111-D1, 123-D6, and so on. To facilitate the description of different configurations, we number these cases and differentiate them according to the position of edge and the level of edge height. In a $3 \times 3$ window, along a column, there are four edge height levels plus one (no edge); see Fig. 1(a). Since there are three columns, the total combinations are $5^{3}=125$. For example, 222 means that there are three edges with height equals 2 in the three columns of the window; see Fig. 1(c). Thanks to the rotation invariance, many cases share the same configuration after rotation (e.g., $90^{\circ}, 180^{\circ}$, and $270^{\circ}$ ). For example, 222 and 333,123 , and 234. Similarly, symmetric cases also share the same configuration. For example, 123 and 321, 123, and 432. All isomorphic (equivalent) configuration codes are grouped and shown in Table I. Therefore, the number of label configurations, $X$, that we need to consider is 20 . This is exactly the number of rows in Fig. 2 (double columns). In addition, it is often not the case that one label configuration of $X$ corresponds to only one boundary configuration because of the difference in edge orientation outside the window. As such, it is necessary to consider these situations. For example, the 111 label configuration 
TABLE II

Using the First-Order Neighborhood System, This Table Lists the Counting Results of All the Preferable MRF $X$ and $D$ Configurations. These Results Show 1) Whether the Center Pixel Belongs to Boundary or Not (SeCond Column), 2) How Many Neighbors Belong to Boundary (Third Column), 3) How Many Neighbors Belong to Different Regions From the

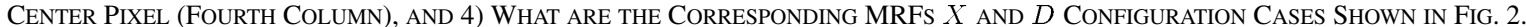
If A Single $X$ Code is Shown, All its $D$ Configurations ARe InCluded. For Example, “212” Means “212D1-D3” (FifTh Column)

\begin{tabular}{|c|c|c|c|l|}
\hline Row number & $d_{i}$ & $\sum_{j \in N_{i}} d_{j}$ & $\sum_{j \in N_{i}} x_{i} \oplus x_{j}$ & MRF X and D configurations \\
\hline 1 & 0 & 0 & 0 & no edge in the window \\
\hline 2 & 0 & 1 & 0 & $111 \mathrm{D} 1$ \\
\hline 3 & 0 & 2 & 0 & $111 \mathrm{D} 2 \mathrm{D} 3,112 \mathrm{D} 1 \mathrm{D} 4$ \\
\hline 4 & 0 & 3 & 0 & $111 \mathrm{D} 4 \mathrm{D} 6,112 \mathrm{D} 2 \mathrm{D} 3 \mathrm{D} 5 \mathrm{D} 6,212$ \\
\hline 5 & 0 & 4 & 0 & double edges in the window \\
\hline 6 & 0 & 4 & 1 & double edges in the window \\
\hline 7 & 1 & 1 & 1 & $121 \mathrm{D} 1$ \\
\hline 8 & 1 & 2 & 1 & $113 \mathrm{D} 1,121 \mathrm{D} 2 \mathrm{D} 3,122 \mathrm{D} 1 \mathrm{D} 6$ \\
\hline 9 & 1 & 3 & 1 & $113 \mathrm{D} 2 \mathrm{D} 3,121 \mathrm{D} 4-6,122 \mathrm{D} 2-5,213,222,114 \mathrm{D} 1$ \\
\hline 10 & 1 & 4 & 1 & $114 \mathrm{D} 2 \mathrm{D} 3,143$ \\
\hline 11 & 1 & 2 & 2 & $123 \mathrm{D} 1 \mathrm{D} 4$ \\
\hline 12 & 1 & 3 & 2 & $123 \mathrm{D} 2 \mathrm{D} 3 \mathrm{D} 5 \mathrm{D} 6,223,313,124 \mathrm{D} 1 \mathrm{D} 4$ \\
\hline 13 & 1 & 4 & 2 & $133,141,142,124 \mathrm{D} 2 \mathrm{D} 3 \mathrm{D} 5 \mathrm{D} 6$ \\
\hline 14 & 1 & 3 & 3 & 232 \\
\hline 15 & 1 & 4 & 3 & 131,132 \\
\hline
\end{tabular}

\begin{tabular}{|c|c|c|c|c|c|c|c|c|c|c|c|c|c|c|}
\hline & $\begin{array}{c}x \\
\text { a a a } \\
\text { a a } a \\
\text { a a } a \\
\end{array}$ & $\begin{array}{lll} & & \text { D1 } \\
0 & 0 & 0 \\
0 & 0 & 0 \\
1 & 1 & 1 \\
\end{array}$ & $\begin{array}{rlr} & \mathrm{D} 2 \\
0 & 0 & 0 \\
1 & 0 & 0 \\
1 & 1 & 1 \\
\end{array}$ & 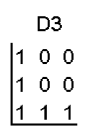 & 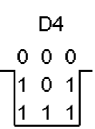 & $\begin{array}{c}\text { D5 } \\
-\left|\begin{array}{lll}1 & 0 & 0 \\
1 & 0 & 1 \\
1 & 1 & 1\end{array}\right|\end{array}$ & $\begin{array}{l}\text { D6 } \\
\left|\begin{array}{lll}1 & 0 & 1 \\
1 & 0 & 1 \\
1 & 1 & 1\end{array}\right|\end{array}$ & $\begin{array}{c}\mathrm{X} \\
\mathrm{a} a \mathrm{a} \\
123 \mathrm{a} a \sqrt{\mathrm{b}} \\
\mathrm{a} \sqrt{\mathrm{b}} \mathrm{b}\end{array}$ & $\begin{array}{lll} & \text { D1 } \\
0 & 0 & 1 \\
0 & 1 & 1 \\
1 & 1 & 0\end{array}$ & 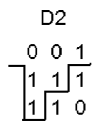 & $\begin{array}{c}\text { D3 } \\
\mid \begin{array}{lll}1 & 0 & 1 \\
1 & 1 & 1 \\
1 & 1 & 0\end{array}\end{array}$ & \begin{tabular}{lll} 
& \multicolumn{2}{c}{$\mathrm{D} 4$} \\
0 & 0 & 1 \\
0 & 1 & 1 \\
1 & 1 & 1
\end{tabular} & 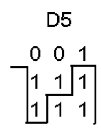 & $\begin{array}{c}\text { D6 } \\
\left|\begin{array}{lll}1 & 0 & 1 \\
1 & 1 & 1 \\
1 & 1 & 1\end{array}\right|\end{array}$ \\
\hline & 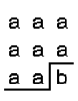 & $\begin{array}{lll}0 & 0 & 0 \\
0 & 0 & 1 \\
1 & 1 & 1 \\
\end{array}$ & ]$_{0}^{0} \begin{array}{lll}1 & 0 & 0 \\
1 & 0 & 1 \\
1 & 1 & 1\end{array}$ & $\mid \begin{array}{lll}1 & 0 & 0 \\
1 & 0 & 1 \\
1 & 1 & 1\end{array}$ & $\begin{array}{lll}0 & 0 & 1 \\
0 & 0 & 1 \\
1 & 1 & 1 \\
\end{array}$ & $-\begin{array}{lll}0 & 0 & 1 \\
1 & 0 & 1 \\
1 & 1 & 1\end{array}$ & {$\left[\begin{array}{lll}1 & 0 & 1 \\
1 & 0 & 1 \\
1 & 1 & 1\end{array}\right.$} & $\begin{array}{r}\text { a a a } \\
131 \text { a } \\
\text { a } \\
\text { a }\end{array}$ & \begin{tabular}{l|l|l}
0 & 1 & 0 \\
1 & 1 & 1 \\
1 & 1 & 1 \\
\end{tabular} & $\mid \begin{array}{lll}1 & 1 & 0 \\
1 & 1 & 1 \\
1 & 1 & 1 \\
& \end{array}$ & $\left|\begin{array}{lll}1 & 1 & 1 \\
1 & 1 & 1 \\
1 & 1 & 1\end{array}\right|$ & & & \\
\hline & $\begin{array}{lll}a & a & a \\
a & a & b \\
a & a & b\end{array}$ & $\begin{array}{lll}0 & 0 & 1 \\
0 & 1 & 1 \\
1 & 1 & 1 \\
\end{array}$ & 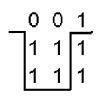 & $\mid \begin{array}{lll}1 & 0 & 1 \\
1 & 1 & 1 \\
1 & 1 & 1\end{array}$ & & & & 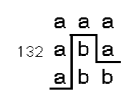 & \begin{tabular}{l|l|l}
0 & 1 & 0 \\
1 & 1 & 1 \\
1 & 1 & 1
\end{tabular} & $\begin{array}{lll}1 & 1 & 0 \\
1 & 1 & 1 \\
1 & 1 & 1\end{array}$ & {$\left[\begin{array}{lll}1 & 1 & 1 \\
1 & 1 & 1 \\
1 & 1 & 1\end{array} \mid\right.$} & \begin{tabular}{l|l|l}
0 & 1 & 1 \\
1 & 1 & 1 \\
1 & 1 & 1
\end{tabular} & & \\
\hline & $\begin{array}{l}a \text { a } a \\
\text { a } a \text { a } \\
\text { a b } a\end{array}$ & $\begin{array}{lll}0 & 0 & 0 \\
0 & 1 & 0 \\
1 & 1 & 1 \\
1 & 1\end{array}$ & 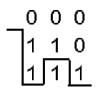 & $\begin{array}{lll}1 & 0 & 0 \\
1 & 1 & 0 \\
1 & 1 & 1\end{array}$ & {$\left[\begin{array}{ccc}0 & 0 & 0 \\
1 & 1 & 1 \\
1 & 1 & 1\end{array}\right]$} & $-\left[\begin{array}{lll}1 & 0 & 1 \\
1 & 1 & 1 \\
1 & 1 & 1\end{array} \mid\right.$ & $\left|\begin{array}{lll}1 & 0 & 0 \\
1 & 1 & 1 \\
1 & 1 & 1\end{array}\right|$ & 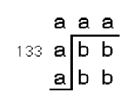 & \begin{tabular}{l|ll}
0 & 1 & 1 \\
1 & 1 & 1 \\
1 & 1 & 0
\end{tabular} & $\mid \begin{array}{lll}1 & 1 & 1 \\
1 & 1 & 1 \\
1 & 1 & 0\end{array}$ & {$\left[\begin{array}{lll}1 & 1 & 1 \\
1 & 1 & 1 \\
1 & 1 & 1\end{array} \mid\right.$} & $\begin{array}{lll}0 & 1 & 1 \\
1 & 1 & 1 \\
1 & 1 & 1\end{array}$ & & \\
\hline & 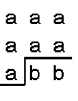 & 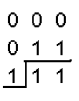 & 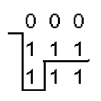 & $\begin{array}{lll}1 & 0 & 0 \\
1 & 1 & 1 \\
1 & 1 & 1\end{array}$ & $\left|\begin{array}{lll}1 & 0 & 1 \\
1 & 1 & 1 \\
1 & 1 & 1\end{array}\right|$ & 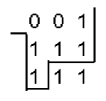 & $\begin{array}{lll}0 & 0 & 1 \\
0 & 1 & 1 \\
1 & 1 & 1\end{array}$ & 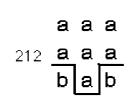 & $\begin{array}{lll}0 & 0 & 0 \\
1 & 0 & 1 \\
1 & 1 & 1\end{array}$ & $\begin{array}{l}\mid \begin{array}{lll}1 & 0 & 0 \\
1 & 0 & 1 \\
1 & 1 & 1\end{array}\end{array}$ & 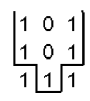 & & & \\
\hline & $\begin{array}{l}\text { a } a \text { a } \\
\text { a } a b^{b} \\
b \\
b\end{array}$ & $\begin{array}{lll}0 & 0 & 1 \\
1 & 1 & 1 \\
1 & 1 & 1 \\
1 & 1 & 1\end{array}$ & 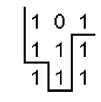 & & & & & 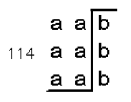 & \begin{tabular}{ll|l}
0 & 1 & 1 \\
0 & 1 & 1 \\
1 & 1 & 1 \\
\end{tabular} & 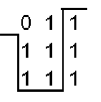 & {$\left[\begin{array}{ll|l}1 & 1 & 1 \\
1 & 1 & 1 \\
1 & 1 & 1 \\
\end{array}\right.$} & & & \\
\hline & $\begin{array}{l}a \text { a } a \\
a \quad a \quad a \\
b \quad b \quad b\end{array}$ & $\begin{array}{lll}0 & 0 & 0 \\
1 & 1 & 1 \\
1 & 1 & 1\end{array}$ & 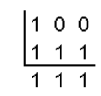 & $\frac{\left|\begin{array}{lll}1 & 0 & 1 \\
1 & 1 & 1\end{array}\right|}{1}$ & & & & \begin{tabular}{rrr|r} 
& $\mathrm{a}$ & $\mathrm{b}$ & $\mathrm{a}$ \\
141 & $\mathrm{a}$ & $\mathrm{b}$ & $\mathrm{a}$ \\
& $\mathbf{a}$ & $\mathrm{b}$ & $\underline{\mathrm{a}}$ \\
\end{tabular} & \begin{tabular}{l|l|l}
1 & 1 & 1 \\
1 & 1 & 1 \\
1 & 1 & 1 \\
\end{tabular} & & & & & \\
\hline & $\begin{array}{l}a \quad a \quad a \\
a \quad a b \\
b b \quad b \quad b\end{array}$ & $\begin{array}{lll}0 & 0 & 1 \\
1 & 1 & 1 \\
1 & 1 & 0\end{array}$ & 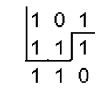 & $\begin{array}{lll}0 & 0 & 1 \\
1 & 1 & 1 \\
1 & 1 & 1\end{array} \mid$ & $\frac{\left|\begin{array}{lll}1 & 0 & 1 \\
1 & 1 & 1 \\
1 & 1 & 1\end{array}\right|}{\mid}$ & & & 142 \begin{tabular}{ll|l|l} 
& $a$ & $b$ & $a$ \\
& $a$ & $b$ & $b$ \\
$b$ & $b$ & $b$
\end{tabular} & \begin{tabular}{l|l|l}
1 & 1 & 1 \\
1 & 1 & 1 \\
1 & 1 & 1
\end{tabular} & & & & & \\
\hline & 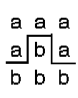 & $\begin{array}{lll}0 & 1 & 0 \\
1 & 1 & 1 \\
1 & 0 & 1\end{array}$ & $\begin{array}{lll}1 & 1 & 0 \\
1 & 1 & 1 \\
1 & 0 & 1\end{array}$ & $\begin{array}{lll}1 & 1 & 1 \\
1 & 1 & 1\end{array} \mid$ & & & & \begin{tabular}{rr|rl} 
& $a$ & $b$ & $a$ \\
143 & $a$ & $b$ & $b$ \\
$a$ & $a$ & $b$ & $b$
\end{tabular} & \begin{tabular}{l|ll}
1 & 1 & 1 \\
1 & 1 & 1 \\
1 & 1 & 0
\end{tabular} & \begin{tabular}{l|l|l}
1 & 1 & 1 \\
1 & 1 & 1 \\
1 & 1 & 1
\end{tabular} & & & & \\
\hline & $\begin{array}{l}a \\
a \\
b \\
b \\
b\end{array}$ & \begin{tabular}{l|l|l}
1 & 0 & 1 \\
1 & 1 & 1 \\
1 & 1 \\
1 & 1
\end{tabular} & & & & & & 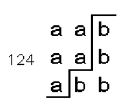 & 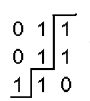 & \begin{tabular}{ll|l}
0 & 1 & 1 \\
1 & 1 & 1 \\
1 & 1 & 0
\end{tabular} & $\mid \begin{array}{lll}1 & 1 & 1 \\
1 & 1 & 1 \\
1 & 1 & 0\end{array}$ & \begin{tabular}{lll|}
0 & 1 & 1 \\
0 & 1 & 1 \\
1 & 1 & 1
\end{tabular} & {$\left[\begin{array}{lll}0 & 1 & 1 \\
1 & 1 & 1 \\
1 & 1 & 1\end{array} \mid\right.$} & $\left|\begin{array}{lll}1 & 1 & 1 \\
1 & 1 & 1 \\
1 & 1 & 1\end{array}\right|$ \\
\hline
\end{tabular}

Fig. 2. Considering a single edge passing through a $3 \times 3$ window, this figure shows all the desirable configurations of MRFs $X$ and $D$ in the window. One configuration of label MRF $X$ can correspond to more than one configuration of boundary MRF $D$, e.g., $D 1, D 2, \ldots, D 6$.

$X$ has six corresponding boundary configurations (each having the same configuration in the $3 \times 3$ window but a different edge orientation outside the window), D1, D2, .., D6 (see left top row in Fig. 2), and 113 has three (see left third row in Fig. 2).
There are two points worth noticing with regard to the configurations shown in Fig. 2. First, we do not show the scenarios where there are no edges in some columns, i.e., $0 \mathrm{XX}, \mathrm{XOX}$, and $\mathrm{XX} 0$. We find that when it comes to the energy formulation, 
each of these scenarios is equivalent to one of the cases listed in Fig. 2, e.g., after a $90^{\circ}$ clockwise rotation, 012 is the same as 211. This is also equivalent to 112 in Fig. 2 because of the symmetry so we do not show them in the figure for the sake of brevity. Second, multiple edges can appear in a $3 \times 3$ window (but not often) and, we do not show them in Fig. 2 for the reason that they can be equivalent to the single-edge situations in terms of energy formulation (e.g., the case where two straight, horizontal edges pass through a $3 \times 3$ window, that is, a combination of configurations 222 and 333, shares the same energy formulation as the case of row 13 in Table II). This makes the presentation more concise. Note that it is very complicated to consider all the possible scenarios of multiple edge configurations. There can be 24 edge segments altogether in a $3 \times 3$ window, including the 12 edge segments surrounding the window. Therefore, theoretically, there are $2^{24}$ (over 16 million) combinations of the multiple edge configuration in the $3 \times 3$ neighborhood. These include both the preferred cases and unreasonable cases. In this paper, we mainly focus on the single edge scenarios to simplify the problem.

Once we know which configurations of label MRF $X$ and boundary MRF $D$ should be chosen and how they should be matched, it is not difficult to count the number of labels different from the center pixel $x_{i}$ using $\sum_{j \in N_{i}} x_{i} \oplus x_{j}$, and count the number of the neighboring boundary pixels using $\sum_{j \in N_{i}} d_{j}$. These are essential for the energy function construction. The results are listed in Table II. Note that although we show $3 \times 3$ window in Fig. 2 to illustrate the preferred configurations, we adopt the first order Ising neighborhood system for MRFs. The correspondence of Table II and Fig. 2 is obvious. For instance, row 3 of the table corresponds to four cases (pairs) in the figure, i.e., 111-D2, 111-D3, 112-D1, and 112-D4. This relation of Table II and Fig. 2 is shown in the last column of Table II.

It is straightforward to convert the tally information in Table II to an energy formulation. Each row in the table corresponds to a term in the energy function and should be assigned a low energy value when the three conditions $\left(d_{i}, \sum_{j \in N_{i}} d_{j}\right.$ and $\sum_{j \in N_{i}} x_{i} \oplus$ $x_{j}$ ) are satisfied. The configurations that are not in the table should be penalized and assigned a high energy value. Furthermore, in the energy function, the three conditions in each row are linked with addition, and those terms representing each row are connected with multiplication. Thus, we get our new prior energy formulation, which is given as

$$
\begin{aligned}
U(X, D)= & \sum_{i \in S} \gamma \cdot\left[\delta\left(d_{i}\right) T_{1}(i)+\delta\left(d_{i}-1\right) T_{2}(i)\right], \gamma>0 \\
T_{1}(i)= & \sum_{j \in N_{i}} x_{i} \oplus x_{j} \cdot\left(\left|\sum_{j \in N_{i}} x_{i} \oplus x_{j}-1\right|+\left|\sum_{j \in N_{i}} d_{j}-4\right|\right) \\
T_{2}(i)= & \left(\left|\sum_{j \in N_{i}} x_{i} \oplus x_{j}-1\right|+\prod_{k=1}^{4}\left|\sum_{j \in N_{i}} d_{j}-k\right|\right) \\
& \cdot\left(\left|\sum_{j \in N_{i}} x_{i} \oplus x_{j}-2\right|+\prod_{k=2}^{4}\left|\sum_{j \in N_{i}} d_{j}-k\right|\right) \\
& \cdot\left(\left|\sum_{j \in N_{i}} x_{i} \oplus x_{j}-3\right|+\prod_{k=3}^{4}\left|\sum_{j \in N_{i}} d_{j}-k\right|\right)
\end{aligned}
$$

where $\oplus$ represents the "exclusive or" operation and $\gamma$ is the penalty. Terms $T_{1}$ and $T_{2}$ account for nonboundary $\left(d_{i}=0\right)$ and boundary $\left(d_{i}=1\right)$ situations, respectively. If the conditions of any row in Table II are satisfied, $T_{1}$ or $T_{2}$ becomes zero and a low prior energy is obtained. Originally, there should be 15 terms in the energy formula. It changes into this form because some terms can be combined. Here, we set the same weight to all preferred cases, but we can also assign different importance to each individual case. For example, if we believe that smooth boundaries are more likely, we can set a higher penalty $(\gamma)$ to those terms of 111 and 222 than those of 141 and 414. This makes the model more flexible for different applications.

The motivation to use this model is that a true boundary or edge without noise should be continuous at least in a small window, like a $3 \times 3$ neighborhood. If one site is corrupted by noise and regarded as a boundary, probably the neighborhood would not conform to the true pattern of a reasonable boundary because of the randomness of noise. Then, due to the penalty given by the prior energy term, this case would most likely be discarded. Moreover, this model is capable of keeping complex boundary information because it is tolerant of all kinds of boundary shapes of true objects.

\section{EXPERIMENTAL RESULTS}

In this section, we perform a series of segmentation experiments on synthetic images, simulated medical images and real clinical images. Most of the datasets have complex boundaries and considerable levels of noise, and, thus, result in challenging segmentation tasks. We apply the proposed model to both binary and multiclass segmentations, and compare the results with three other existing MRF models in the literature: the multilevel logistic (MLL) model [8], [9] and two types of MRF models with line process (LP) [1], [3].

\section{A. Parameter Estimation}

There are a few parameters related to the proposed method needed to be estimated before we perform segmentation. These parameters are the mean intensity $\mu_{j}$ for every region $j$ [in (7)], the standard deviation $\sigma$ of the noise [in (7)], the threshold for the gradient map and the weight, $\gamma$, of the prior energy [in (11)].

There are usually two ways to estimate the mean intensity $\mu_{j}$ of each region $j$. One is the supervised method which requires a user to select a region of interest (ROI) inside each region and calculate the mean of the ROI intensity. The assumption is that a small part (the aforementioned ROI) of the image is labeled manually. Another way is the unsupervised method [16]. The estimation can be performed by some statistical approaches such as simultaneous segmentation and estimation [9], or the expectation minimization (EM) algorithm [11]. These methods are often related to the analysis of the image intensity statistics, e.g., histograms. The unsupervised approach helps to make the segmentation method more automatic because it does not need manual initialization or training data [16]. However, the unsupervised methods are usually not as accurate as the supervised ones because the formers face more challenges such as estimation and segmentation can result in a chicken-and-egg dilemma 
[9]. Therefore, in the experiments, we adopt the supervised estimation of the means for both the proposed model and the control models because manual segmentations are available. Similarly, we estimate the standard deviation of the noise using the supervised method. As such, the likelihood model in (7), which is not the main contribution of this paper, can be as accurate as possible.

We also need to choose a global threshold in the gradient map of the input image so that the line process in the control models or the boundary MRF $D$ in the proposed model can be initialized. The requirement for setting the threshold is that the boundary between different regions should be recognized as accurately as possible. One estimation method is to set the global threshold comparable to the minimum of all the absolute differences of mean intensity between two distinct neighboring regions in the intensity image (i.e., $\min _{i, j}\left|\mu_{i}-\mu_{j}\right|$, where regions $i$ and $j$ are adjacent). Since the mean intensity for each region has been already estimated, the estimation of the global threshold can be achieved.

The parameter $\gamma$ balances the importance between the prior energy and the likelihood energy. In our previous work [17], we used trial and error to estimate the optimal weight by trying on different values individually. Other work adopted more automatic methods, e.g., generalized cross validation method or standard regularization method [3]. All these approaches can be used in the proposed method. In the current experiments, we set the initial value of $\gamma$ based on the mean intensity of all the regions and the noise level of the given input image from the simulated or real datasets. The initial value of $\gamma$ is set based on a measure, $M E=\min _{i, j}\left(\left|\mu_{i}-\mu_{j}\right|\right) / \sigma(i, j$ refer to any two region numbers). This measure gives the ratio of the smallest absolute difference between the mean intensity in two regions to the noise level. Note that the means and standard deviation have already been estimated. If ME is relatively low (i.e., lower than 4.5), the image is relatively noisy or it contains regions having relatively close intensity values. As such, we set the initial value of $\gamma$ to 15 in order to attach more weight to the prior energy and impose more prior constrain. If $\mathrm{ME}$ is relatively high (i.e., not lower than 4.5), the image is relatively clean or the regions inside the image have relatively large intensity gaps. Therefore, we set the initial value of $\gamma$ to 5 in order to give less weight associated with the prior energy and trust the likelihood energy more. We then perform searching around the initial value within a small integer range [initial -5 , initial +5 ] and pick up the value that makes the model work best (i.e., with the lowest error rate).

\section{B. Synthetic Images}

We first test and compare the segmentation performance of the three existing MRF models and our model using some synthetic data including bars and circles with different widths in different images. Thick bars or circles in the synthetic images are around six-pixel wide while thin ones are three-pixel wide. The image size is $128 \times 128$ pixels. However, in order to test images with complex boundaries, we do not generate smooth bars and circles with regular boundaries and we make the

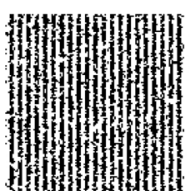

(a)

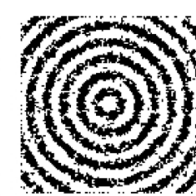

(b)

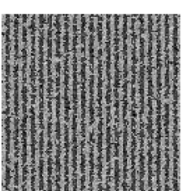

(c)

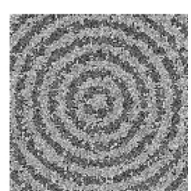

(d)
Fig. 3. Some examples of the synthetic images. Subfigures (a) and (b) are the true patterns of bar and circle images, respectively. Subfigures (c) and (d) are the corresponding noisy images with $\mathrm{SNR}=3$ and 2 , respectively.

boundaries bumpy instead. The bumpiness of the boundary is generated by randomly perturbing the originally straight and smooth boundary with a small shift along the normal direction (the direction can be either inward or outward). The range of the perturbation is within two pixels. For example, at a boundary pixel, if the randomly generated offset is +2 , the boundary on this pixel is shifted along the positive normal direction by two pixel length. If the offset is -1 , the boundary is shifted along the negative normal by a pixel. We repeat this random operation everywhere on the boundary. Then, the original smooth boundary is converted to a bumpy shape. Moreover, the inner regions of objects and background are interspersed with square holes (with 1 to 2 pixels in dimension). These holes are also randomly distributed; see Fig. 3 (a) and (b) for two examples, whose objects are bars and circles, respectively. These images serve as the ground truth for validation of methods. The objects are high in intensity and the background is dark. We then corrupt each synthetic image with a Gaussian noise having zero mean and the same standard deviation (SD) following the style in our previous work [17] [see Fig. 3(c) and (d)]. The SNR is calculated by $\left(\mu_{o}-\mu_{b}\right) / \sigma$, where $\mu_{o}$ and $\mu_{b}$ are the true intensities of object and background regions, respectively, and $\sigma$ represents the SD of the added noise. In the experiments, $\mu_{o}=160$ and $\mu_{b}=100$. The above steps lead to a difficult binary segmentation task.

To evaluate the performance of the proposed model, we compare it with three existing MRF models using these synthetic images. The first model uses a single label MRF, $X$, for the segmentation and has the same prior energy definition without bias field as in [8] (hereafter referred to as MLL). This is the widely used multilevel logistic (MLL) model [9]. The second model, which is derived from [1], makes use of the line process (hereafter referred to as LP1). The third model [3] also adopts the line process but with different interactions between discontinuity sites (hereafter referred to as LP2). The likelihood function (7) is the same for these three MRF models and our model. The difference between these models and ours lies in the prior energy function which embodies the interactions between sites. The prior functions of the first [MLL, (12)], second [LP1, (13)], and third [LP2, (14)] models are given, respectively, in (12)-(14), shown at the bottom of the next page. Here, if $x=0, \delta(x)=1$; else $\delta(x)=0 . N_{i}^{v}$ and $N_{i}^{h}$ are the sets of neighbors in vertical and horizontal directions, respectively. Equation (12) corresponds to the [8, Eq. (3)], and the potential function $V(x, y)=$ 0 if $x=y$; else, $V(x, y)=1$.

The second prior energy, LP1, as described in (13), is derived according to the Geman and Geman's paper [1, right column, 
fifth and sixth paragraphs on p. 732]. Equation (13) is slightly different from the description in their paper in that we introduce the cost of inserting an edge between two adjacent pixels (while [1] did not consider). This makes the model more flexible. For instance, if the sites $i$ and $j$ have different labels $x_{i}$ and $x_{j}$, it is more preferable to insert a vertical edge with the cost of $\gamma^{v}$. $\gamma^{v}$ and $\gamma^{h}$ are small constants, which are significantly smaller than 1. In the experiments, we set them to 0.1 . When $\gamma^{v}$ and $\gamma^{h}$ equal 0 , this model reduces to Gemans' model. Similar style of line process formulation was also adopted in $[9$, Sec. 2.3.1, Eq. (2.40) ]. The last term of (13), $U(H, V)$, is illustrated in [1, Fig 5(a)]. It assigns potentials to various patterns of line process.

Equation (14) corresponds to [3, Eqs. (27) and (28)]. $a$ and $b$ represent the $x, y$ coordinates of the image lattice. The only difference lies in that the reference paper [3] used a piecewise continuous model for surface reconstruction and we use a piecewise constant model for image segmentation.

We optimize all four MRF formulations using the iterated conditional modes (ICM) algorithm [2]. ICM is a general simple algorithm which is gradient-based. Simultaneously, ICM allows only one label to be changed and the label making the lowest energy is chosen. This is repeated at every site. Then, one iteration of ICM is completed. When no change of one site label can make the energy further decrease, the algorithm comes to convergence. The pseudo-code of the ICM algorithm for the proposed model is shown in Algorithm 1. This framework is suitable for the other three models if we replace the prior energy function $U_{\text {prior }}$ with corresponding forms (e.g., (12) but without the summation over every pixel). $U_{\text {likelihood }}$ and $U_{\text {prior }}$ in (15) should include all the terms in the total energy that may change due to the change of $x_{i}$ and $d_{i}$. In the experiments, MaxItn is set to 10
Algorithm 1. The pseudo-code for the iterated conditional modes algorithm used for the proposed MRF model.

Step 1: Let $k$ be the iteration index and set $k=1$.

Step 2: Let $i$ be site index and set $i=1$.

Step 3: Compute (15), shown at the bottom of the page, where $U_{\text {likelihood }}\left(y_{i} \mid x_{i}^{k}\right)=\left(y_{i}-\mu_{x_{i}^{k}}\right)^{2} / 2 \sigma^{2}$ and

$$
\begin{aligned}
U_{\text {prior }}\left(x_{i}^{k}, d_{i}^{k} \mid x_{N_{i}}^{k-1}, d_{N_{i}}^{k-1}\right) & \\
& =\gamma \cdot \sum_{j \in N_{i} \cup\{i\}}\left[\delta\left(d_{j}\right) T_{1}(j)+\delta\left(d_{j}-1\right) T_{2}(j)\right]
\end{aligned}
$$

[functions $T_{1}, T_{2}$ are defined in (11)].

Step 4: If $i=n$, then go to Step 5; else, $i=i+1$ and go to Step 3. $n$ is the total number of sites.

Step 5: If $k=$ MaxItn or $x_{i}^{k}=x_{i}^{k-1}, d_{i}^{k}=d_{i}^{k-1}$, for every $i \in\{1, \ldots, n\}$, then go to Step 6; else, $k=k+1, i=1$ and go to Step 3. MaxItn is the maximum number of iterations.

Step 6: Final estimate $\hat{x}_{i}=x_{i}^{k}, i=1, \ldots, n$.

Since we know the ground truth of the synthetic images, it is convenient to quantify the performance of each segmentation method by calculating the error rate. The error rate of the segmentation is given by

$$
\frac{\text { Number of misclassified pixels }}{\text { Total number of pixels in the image }} \times 100 \text {. }
$$

The initial configuration of the label MRF $X$ is obtained by using the maximum likelihood estimate [9], $X^{(0)}=\arg \max _{X \in \Omega_{X}} P(Y \mid X)$, and the initial configuration of the boundary MRF $D$ can be set by the methods stated in

$$
\begin{aligned}
\text { For MLL, } U(X)= & \sum_{i \in S} \sum_{j \in N_{i}} V\left(x_{i}, x_{j}\right) \\
\text { For LP1, } U(X, H, V)= & \sum_{i \in S} \sum_{j \in N_{i}^{v}}\left[\left(1-\delta\left(x_{i}-x_{j}\right)\right)\left(1-v_{i, j}\right)+\gamma^{v} v_{i, j}\right] \\
& +\sum_{i \in S} \sum_{k \in N_{i}^{h}}\left[\left(1-\delta\left(x_{i}-x_{k}\right)\right)\left(1-h_{i, k}\right)+\gamma^{h} h_{i, k}\right]+U(H, V) \\
\text { For LP2, } U(X, H, V)= & \sum_{a, b}\left[\left(1-\delta\left(x_{a, b}-x_{a, b-1}\right)\right)\left(1-v_{a, b}\right)+\gamma v_{a, b}\left(1-0.5\left(v_{a, b-1}+v_{a, b+1}\right)\right)\right] \\
& +\sum_{a, b}\left[\left(1-\delta\left(x_{a, b}-x_{a-1, b}\right)\right)\left(1-h_{a, b}\right)+\gamma h_{a, b}\left(1-0.5\left(h_{a-1, b}+h_{a+1, b}\right)\right)\right]
\end{aligned}
$$

$$
\left(x_{i}^{k}, d_{i}^{k}\right)=\arg \min _{x_{i}^{k} \in\{0, \ldots, m-1\}, d_{i}^{k} \in\{0,1\}}\left(U_{\text {likelihood }}\left(y_{i} \mid x_{i}^{k}\right)+U_{\text {prior }}\left(x_{i}^{k}, d_{i}^{k} \mid x_{N_{i}}^{k-1}, d_{N_{i}}^{k-1}\right)\right)
$$




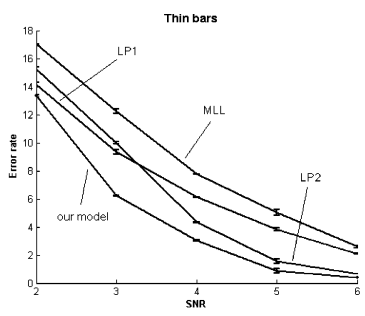

(a)

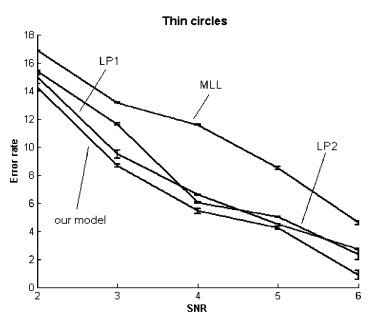

(c)

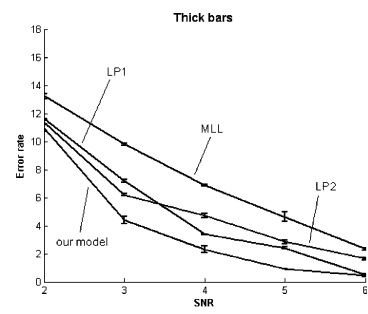

(b)

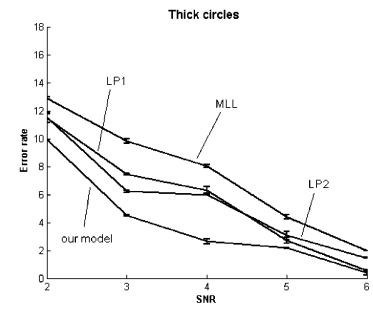

(d)
Fig. 4. Error rates of the three existing MRF models and our model over various SNRs.

Section III-A. All parameters in the likelihood functions are known for the synthetic images.

We repeat each experiment five times (with regenerated random noise), calculate the means and SDs, and plot error rates of four methods over different values of SNR in Fig. 4. As expected, when the noise level goes up, the segmentation errors of all models increase. For SNRs from 2 to 6, our model can increase the segmentation accuracy by 1 to 8 percentage points, as compared with the other three MRF models. The MLL model always has the highest segmentation error rates and the two LP models (LP1 and LP2) give comparable results to each other. The proposed model always obtain the lowest segmentation errors because its segmented results are closer to the ground truth, as quantitatively shown in Fig. 4.

\section{Simulated Medical Images}

We then apply the proposed model together with the three existing control MRF models to a series of simulated medical images. These brain images are obtained from the simulated brain database, BrainWeb (available online [18]). The database contains a volume of brain magnetic resonance (MR) images with known anatomical labels to each voxel. We select a 2-D slice (the 90th slice) with different levels of noise from the database and perform multiclass segmentation on those images. We divide all brain image pixels into four classes, [gray matter (GM), white matter (WM), cerebrospinal fluid (CSF), and others]. The datum on which we perform tissue classification is a T1-weighted MR image and has $181 \times 217$ pixels. We assign zero intensity to those tissues belonging to "others" (e.g., fat, skin, skull, etc.) and focus on the segmentation of GM, WM, and CSF, because these three tissues occupy most of the brain volume and are characterized by complex structures. Since we know the true tissue label assigned to each pixel, it is easy to estimate the parameters in the likelihood energy function. Here, we set the mean intensities for "others," CSF, GM, and WM to $0,58,117,156$, respectively. BrainWeb database offers four

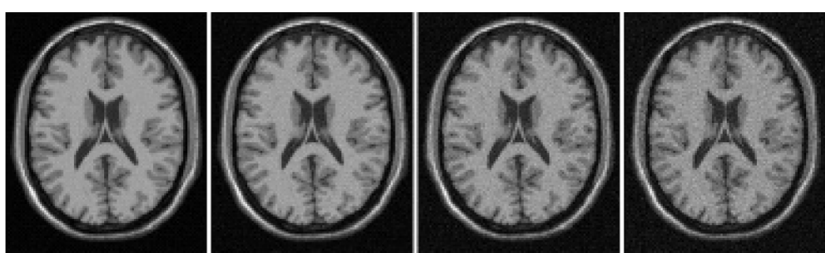

Fig. 5. Ninetieth slice of the BrainWeb dataset. From left to right are images with $3 \%, 5 \%, 7 \%$, and $9 \%$ noise levels, respectively.

TABLE III

SEgMENTATION RESUltS ON THE 90TH SLICE OF THE BRAINWEB T1-WeIGHTED IMAGES With DifFERENT NOISE LEVELS. THE LOWEST ERROR RATES ARE Highlighted IN BOLD

\begin{tabular}{|c|c|c|c|c|}
\hline \multirow{2}{*}{ Noise levels } & \multicolumn{4}{|c|}{ Error rates } \\
\cline { 2 - 5 } & MLL & LP1 & LP2 & Our model \\
\hline $3 \%$ & 2.32 & 1.98 & 2.10 & $\mathbf{1 . 6 7}$ \\
\hline $5 \%$ & 3.23 & 3.15 & 3.00 & $\mathbf{2 . 4 4}$ \\
\hline $7 \%$ & 3.82 & 4.58 & 4.13 & $\mathbf{3 . 4 0}$ \\
\hline $9 \%$ & 7.29 & 6.51 & 6.09 & $\mathbf{5 . 9 6}$ \\
\hline
\end{tabular}

image volumes with four different levels of noise, 3\%, 5\%, 7\%, and $9 \%$ (see Fig. 5), and we test them all. The segmentation results in terms of the error rate are listed in Table III. The segmentation accuracy decreases when the images are corrupted by a larger amount of noise. The proposed method achieves more accurate segmentation results with the advantage of 0.3 to 1.3 percentage points over the other three MRF models.

To testify the use of our boundary MRF model for finding closer boundaries to the ground truth, we compare the boundaries obtained by using the four models with the true, known boundaries. Since the MLL model does not contain boundary MRF and the style of line process is different from the proposed model (LP defines discontinuity in between pixels and we define it on pixels), we directly extract the boundary information from the segmented images generated by each model. The closeness to the ground truth is measured by the means of distance to the closest point (DCP) [19]. In detail, we calculate the distance of every point in a set of boundaries from the control models or our model to the closest point in another set of boundary from the ground truth. Then we sum up all values of DCP and regard the summation as the measure of interest, namely boundary difference. Obviously, the smaller this measure is, the closer the two sets of boundaries are. Due to the complexity of the boundaries, we classify the boundaries in each set into different types, e.g., the boundary between GM and WM, the boundary between GM and others, and the boundary between WM and others, etc. In total, there are six types of boundaries for four classes: GM, WM, CSF, and others. In calculation of the boundary difference, we only consider the distance between the boundaries of the same type in order to make the measure more specific and accurate. The results on the BrainWeb data are listed in Table IV, where we observe that the proposed model obtains boundaries closer to the ground truth than the other three MRF models, and the two LP models (LP1, LP2) get closer boundaries than the MLL model. This is also positively relevant to the segmentation results, as listed in Table III. 
TABLE IV

USING THE 90TH SLICE IN THE BRAINWeb T1-Weighted IMAgES WITH DIFFERENT NOISE LEVELS, THIS TABLE LISTS THE RESULTS BASED ON BOUNDARY DIFFERENCE. THE SMALLEST BOUNDARY DIFFERENCES ARE HIGHLIGHTED IN BOLD

\begin{tabular}{|c|c|c|c|c|}
\hline \multirow{2}{*}{ Noise levels } & \multicolumn{4}{|c|}{ Boundary differences from the ground truth } \\
\cline { 2 - 5 } & MLL & LP1 & LP2 & Our model \\
\hline $3 \%$ & 3318.7 & 2878.6 & 2913.2 & $\mathbf{2 3 9 8 . 7}$ \\
\hline $\mathbf{5 \%}$ & 4294.1 & 3123.4 & 3001.0 & $\mathbf{2 7 1 0 . 5}$ \\
\hline $\mathbf{7 \%}$ & 4474.2 & 4889.3 & 4525.6 & $\mathbf{4 2 9 5 . 1}$ \\
\hline $9 \%$ & 6675.0 & 5837.2 & 5503.1 & $\mathbf{4 6 8 5 . 4}$ \\
\hline
\end{tabular}
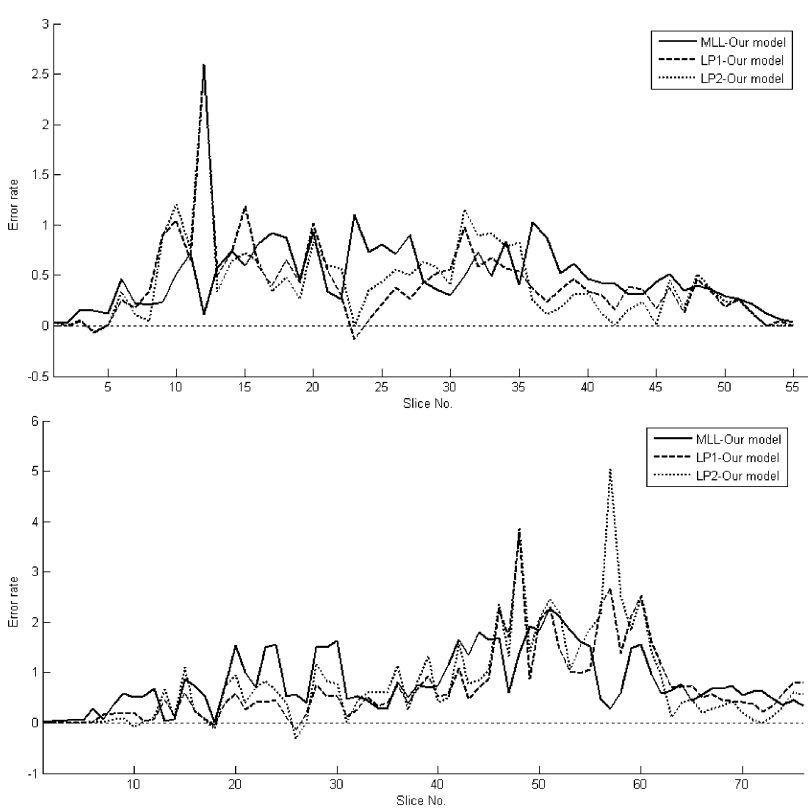

Fig. 6. Segmentation results for an adult male subject brain (top subfigure) and a child subject brain (bottom subfigure). We subtract the error rates of our model from those of the other three models for easy inspection.

\section{Real Clinical Images}

Finally, we apply the four MRF models to the segmentation of real clinical brain images. These real cerebral images are obtained from the Internet Brain Segmentation Repository (IBSR) [20]. IBSR provides manually-guided expert segmentation results along with magnetic resonance brain image data. We test the four MRF models on all brain image slices from two subjects. One is a male subject (56 slices) and the other is a child (76 slices). Both subjects undertook T1-weighted brain scans. The original images have $256 \times 256$ pixels but contain a lot of irrelevant black background regions so we select a rectangular region of interest (ROI) just including the brain volumes in the images. This ROI selection can also make the segmentation more efficient. According to the given ground truth available at IBSR, we classified the image pixels to three classes [gray matter (GM), white matter (WM), and others]. Similar to the experiments on simulated brain volumes, we set the intensity of those pixels belonging to "others" to zero and focus on the segmentation of GM and WM. We estimate the initial model parameters, e.g., mean intensity and standard deviation of each class, based on the true classification of the image volume. The quantitative segmentation results in terms of error rate are shown in Fig. 6, which
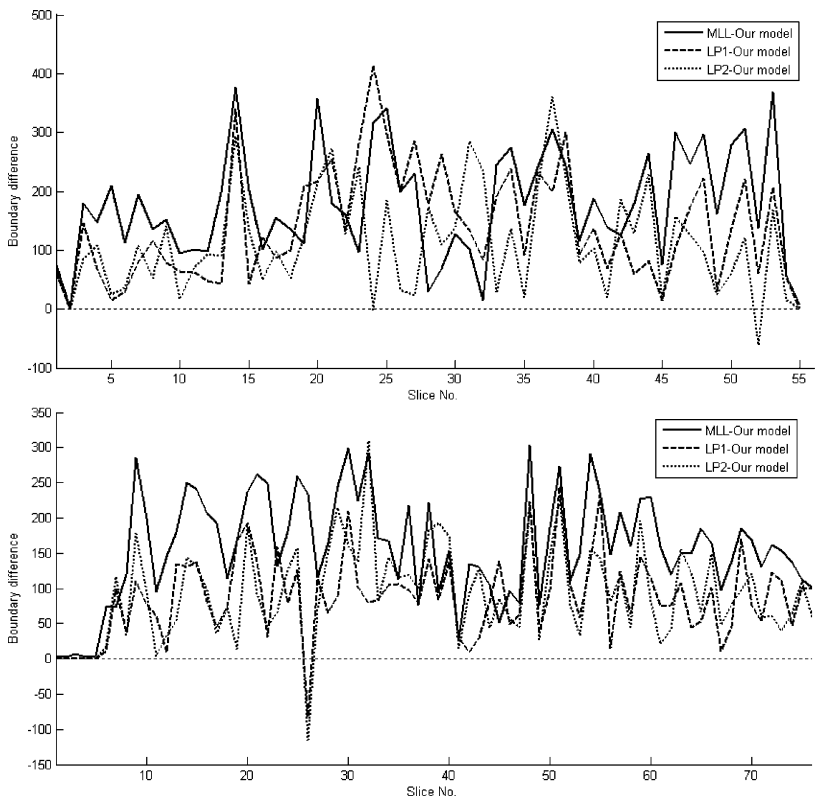

Fig. 7. Boundary evaluation for the four models. The data are the same as in Fig. 6. We subtract the boundary difference of our model from those of the other three models for easy inspection.

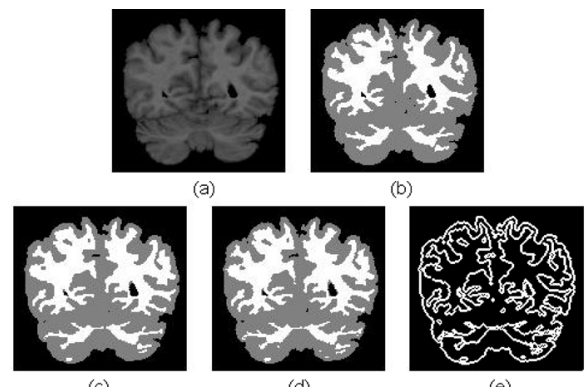

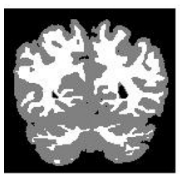

(f)

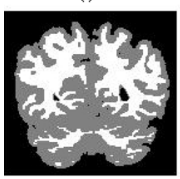

(1)

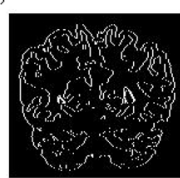

(g)

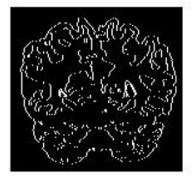

$(k)$

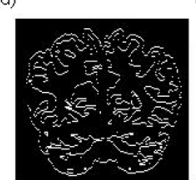

(h)

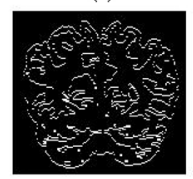

(1)

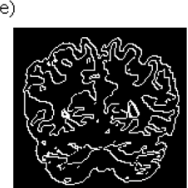

(i)

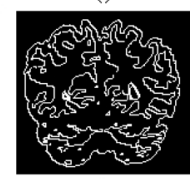

(m)
Fig. 8. Examples of IBSR data and segmentation results. Subfigures (a) and (b) are the images to be segmented and ground truth, respectively. Subfigure (c) is the result of MLL model. Subfigures (d) and (e) are the labeling and boundary results of the proposed model. Subfigures (f)-(i) are the results of labeling, vertical line process, horizontal line process, and combination of vertical and horizontal line process of LP1 model. Subfigures (j)-(m) are the results of labeling, vertical line process, horizontal line process, and combination of vertical and horizontal line process of LP2 model.

plots the results of all slices of both subjects. Notice that we plot the differences between error rates of the controls and our model. More precisely, we subtract the error rates of our model from those of the other three models individually. If the positive value is obtained after the subtraction, this means that the error rate of our model is smaller. As shown in Fig. 6, it is found that the proposed model outperforms the other three models (MLL, 


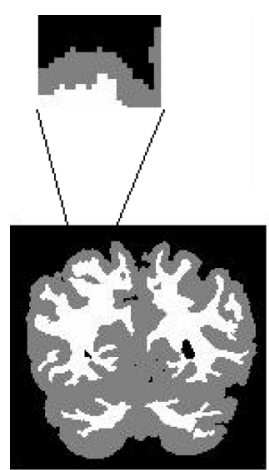

(a)

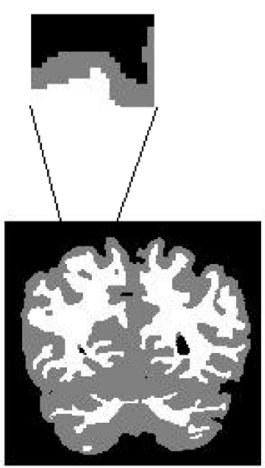

(b)

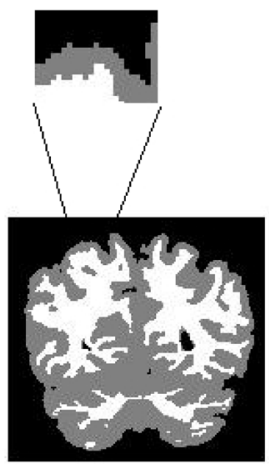

(c)

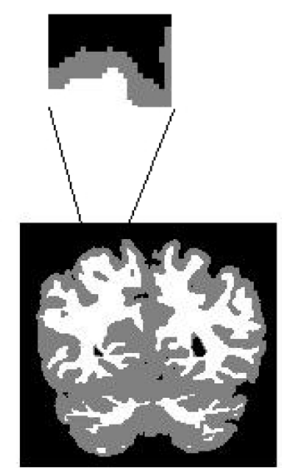

(d)

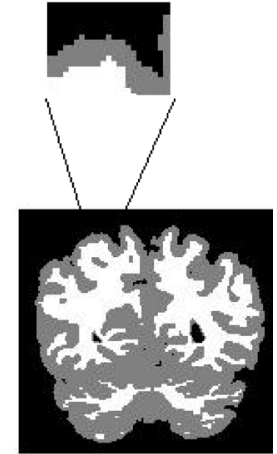

(e)

Fig. 9. Closer checking of the segmentation results near boundaries. Subfigures (a)-(e) are the segmented images obtained by using the ground truth, MLL model, the proposed model, LP1 model and LP2 model, respectively. The first row is the magnified region near boundaries when we zoom in near the top left corner of the brain image.

LP1, LP2) in terms of segmentation accuracy because much of the figure's three curves is above the zero level except for very few exceptions. The two LP models are similar in terms of segmentation accuracy and perform slightly better than the MLL model.

Similar to the experiments in the previous section, we check the boundary difference of the four models using the ground truth. The results on the IBSR data are shown in Fig. 7. In the figure, we calculate and show the difference between boundary differences of other models and ours. We subtract the boundary difference of our model from those of the three other models. As shown in the figure, most parts of the three curves are larger than zero. There are only one or two exceptions. This means that most of the boundary differences between the proposed model and ground truth are less than the boundary differences between the other three models and ground truth. As such, the proposed model can find boundaries closer to the ground truth than the other three MRF models, and LP models get closer boundaries than MLL model in general.

To visualize the difference between the four models, we show some segmentation results in Fig. 8. These results are based on the same brain image volume so that we can visually compare the results obtained by using the four models. We show the segmented images, in which bright regions represent WM, the gray regions represent GM, and the black regions represent other tissue classes or the background. It is observed that the segmentations of image volumes obtained by using the four different MRF models do not differ significantly. However, when we zoom in to look closer at the subtle boundary areas, we can find that the proposed model tends to preserve the boundary shape better than the other MRF models (see Fig. 9, in which our method does not over smooth the boundary). Besides, although the boundary results are not our solitary objective, they can infer the quality of segmentation to some extent. We can see that the proposed method gets better boundaries than the other two line process methods because the former boundaries have less breaks and are closer to ground truth even though the boundaries change subtly.

One observation needs some discussions. Some medical image segmentation results are not completely consistent with our intuition. We believe that the reasons are as follows. The ground truth is generated under the supervision of trained radiologists with prior knowledge and past clinical experiences. We have to trust the "gold standard" when the result conflicts with our observation. For example, in Fig. 8, it appears that there is a part of white matters in the central area, but from the results of all the four segmentation methods, that part is proved to be quite dim and we tend to believe that it belongs to grey matter.

\section{DISCUSSION}

In the previous section, we have performed binary and multiclass segmentations on images with the known ground truth. It is shown through experiments that the proposed method can outperform the three conventional MRF based segmentation methods (MLL, LP1, LP2). Using the boundary MRF $D$, our model has the advantage of directly taking into account the discontinuity between different regions while a single MRF (e.g., MLL) does not have this advantage [8]. If constructed properly, the coupled boundary MRF $D$ would probably help the label MRF $X$ to improve its segmentation performance because more information, in particular boundary information, is incorporated extensively in the segmentation process. The MLL model is a powerful tool that is widely used to perform segmentation based on the assumption that pixels of the same class should cluster. As such, this model strongly prefers one pixel sharing the same label with its neighbors or most of its neighbors. This is reasonable but when it comes to the boundary, the MLL model may try to smooth the discontinuity. This is a major drawback of the MLL model. On the contrary, the proposed model solves the problem satisfactorily by taking into account most of the possibilities when a boundary exists in the window of interest.

The line process was introduced because researchers intended to solve the problem related to discontinuities. This allows and models interactions between the boundary field and the label field but works in a less effective way than the proposed method. The interaction between boundary sites in our model is far more sophisticated than those proposed by Geman and Geman [1] (LP1) and Geiger and Girosi [3] (LP2). We study all the possible scenarios of single edge occurring in a neighborhood window, and the corresponding configurations 
of label and boundary MRFs. Moreover, we couple the two MRFs, $D$ and $X$, more compactly than LP2, which used a simple model, i.e., [3, Eq. (28)]. The work in LP1 only studied six cases of edge configurations. This seems to be insufficient to tackle complicated boundaries. The experimental results (e.g., Fig. 9) infer this point. The boundaries found by the proposed model preserve better shapes than the LP1 and LP2 models. Owing to the consideration of discontinuity, the LP models often find boundaries closer to the ground truth than MLL model.

The contribution of this paper lies in a new formulation of MRF model, but the final result of MRF model will also depend on the MRF solvers. In the comparisons, we use the same solver, i.e., ICM, to find the local optima for all models. ICM algorithm is commonly used and very efficient. However, it is known to be sensitive to initialization. This is the reason why we need to obtain a relatively good starting condition in the initialization. Another limitation of ICM is that it can only change one label at one time and this may be the reason why the proposed model has moderate improvement over the control models. Another reason for the moderate improvement is that boundary is not the majority part of the whole image and the improvement on boundary may not be accompanied by significant improvement of overall segmentation accuracy.

It is still under study whether there are good solvers for MRF models with the line process or the proposed MRF model. Stochastic methods such as the simulated annealing (SA) may be considered because they are more robust to the initializations. In theory, SA can freeze a thermal dynamic system at a global minimum energy level with probability one under the assumption that the system starts at a very high temperature and the cooling schedule is extremely slow. However, there are some limitations related to SA. First, it is not easy to select the initial temperature in SA [21]. Usually, we should select a high enough temperature such that almost all the transitions are accepted in the initial stages of the algorithm. However, setting a too high temperature will elongate the algorithm running time considerably. Second, the choice of cooling schedule of the temperature is tricky. If it is too slow, time complexity is very high. If it is too fast, the final obtained energy can be quite high. Third, and most importantly, the computational cost of SA is unacceptable largely due to the huge configuration space of MRF models on images. For example, when using SA (we implemented the Metropolis sampler [9] in SA, which is known to be more efficient than another popular sampler [22], Gibbs sampler) to solve the simplest MLL model on a binary $128 \times 128$ image, we found that the computation time is several hours on a $1.3-\mathrm{GHz}$ computer with 500-MB RAM. For more complex models (line process or the proposed model) on larger images with multiple classes, it will take even much longer time. This means SA is impractical for the current problem. Therefore, we do not apply SA to all the testing data but apply it to a few examples. The preliminary results show that SA can help the proposed method achieve better results than ICM. Also, the results of the proposed method using SA are better than the other control methods using SA.

We have considered using other deterministic methods besides ICM, e.g., belief propagation [23] or the graph cuts method [24], to solve (approximately) our model, but we are still inves- tigating about how to apply them directly to our model because those methods are only applicable to some specific forms of objective functions [23], [25].

\section{SUMMARY}

In this paper, we have proposed a nontexture segmentation model using compound MRFs based on a boundary model. The main target of this approach is to enhance the performance of segmentation by emphasizing the interactions between label and boundary MRFs. The comparisons with other existing MRF models show that the proposed model can give more accurate segmentation results in both high and low noise level regions while preserving subtle boundary information with high accuracy.

\section{REFERENCES}

[1] S. Geman and D. Geman, "Stochastic relaxation, Gibbs distribution and the Bayesian restoration of images," IEEE Trans. Pattern Anal. Mach. Intell., vol. 6, no. 6, pp. 721-741, Jun. 1984.

[2] J. Besag, "On the statistical analysis of dirty pictures," J. Roy. Statist. Soc. B, vol. 48, pp. 259-302, 1986.

[3] D. Geiger and F. Girosi, "Parallel and deterministic algorithm from MRF's: Surface reconstruction," IEEE Trans. Pattern Anal. Mach. Intell., vol. 13, no. 5, pp. 401-412, May 1991.

[4] F. Jeng and J. Woods, "Compound Gauss-Markov random fields for image estimation," IEEE Trans. Signal Process., vol. 39, no. 3, pp. 683-697, Mar. 1991.

[5] R. Molina, J. Mateos, A. Katsaggelos, and M. Vega, "Bayesian multichannel image restoration using compound Gauss-Markov random fields," IEEE Trans. Image Process., vol. 12, no. 12, pp. 1642-1654, Dec. 2003.

[6] J. Sun, N. Zheng, and H. Shum, "Stereo matching using belief propagation," IEEE Trans. Pattern Anal. Mach. Intell., vol. 25, no. 7, pp. 787-800, Jul. 2003.

[7] F. Arduini, C. Dambra, and C. S. Regazzoni, "A coupled MRF model for sar image restoration and edge-extraction," in Proc. Int. Geosci. Remote Sensing Symp., 1992, vol. 2, pp. 1120-1122.

[8] K. Held, E. Kops, J. Krause, W. Wells, R. Kikinis, and H. MullerGartner, "Markov random field segmentation of brain MR images," IEEE Trans. Med. Imag., vol. 16, no. 6, pp. 878-886, Jun. 1997.

[9] S. Li, Ed., Markov Random Field Modeling in Image Analysis. Tokyo, Japan: Springer-Verlag, 2001.

[10] J. Fwu and P. Djuric, "Unsupervised vector image segmentation by a tree structure-ICM algorithm," IEEE Trans. Med. Imag., vol. 15, no. 6, pp. 871-880, Dec. 1996.

[11] Y. Zhang, M. Brady, and S. Smith, "Segmentation of brain MR images through a hidden Markov random field model and the expectation-maximization algorithm," IEEE Trans. Med. Imag., vol. 20, no. 1, pp. 45-57, Jan. 2001.

[12] M. Woolrich, T. Behrens, C. Beckmann, and S. Smith, "Mixture models with adaptive spatial regularization for segmentation with an application to fmri data," IEEE Trans. Med. Imag., vol. 24, no. 1, pp. 1-11, Jan. 2005.

[13] J. Marroquin, B. Vemuri, S. Botello, E. Calderon, and A. FernandezBouzas, "An accurate and efficient Bayesian method for automatic segmentation of brain MRI," IEEE Trans. Med. Imag., vol. 21, no. 8, pp. 934-945, Aug. 2002.

[14] J. Jimenez-Alaniz, V. Medina-Banuelos, and O. Yanez-Suarez, "Datadriven brain MRI segmentation supported on edge confidence and a priori tissue information," IEEE Trans. Med. Imag., vol. 25, no. 1, pp. 74-83, Jan. 2006.

[15] J. Wu and A. Chung, "A segmentation method using compound Markov random fields based on a general boundary model," in Proc. Int. Conf. Image Processing, 2005, vol. II, pp. 1182-1185.

[16] H. Deng and D. A. Clausi, "Unsupervised image segmentation using a simple MRF model with a new implementation scheme," Pattern Recognit., vol. 37, no. 12, pp. 2323-2335, 2004.

[17] A. C. S. Chung, J. A. Noble, and P. Summers, "Vascular segmentation of phase contrast magnetic resonance angiograms based on statistical mixture modeling and local phase coherence," IEEE Trans. Med. Imag., vol. 23, no. 12, pp. 1490-1507, Dec. 2004. 
[18] BrainWeb, [Online]. Available: http://www.bic.mni.mcgill.ca/ brainweb/

[19] V. Chalana and Y. Kim, "A methodology for evaluation of boundary detection algorithms on medical images," IEEE Trans. Med. Imag., vol. 16, no. 5, pp. 642-652, May 1997.

[20] IBSR, [Online]. Available: http://www.cma.mgh.harvard.edu/ibsr/

[21] P. van Laarhoven and E. Aarts, Eds., Simulated Annealing: Theory and Applications. Dordrecht, The Netherlands: Reidel, 1987.

[22] M. Robini, T. Rastello, and I. E. Magnin, "Simulated annealing, acceleration techniques, and image restoration," IEEE Trans. Image Process., vol. 8, no. 10, pp. 1374-1387, Oct. 1999.

[23] J. S. Yedidia, W. T. Freeman, and Y. Weiss, "Understanding belief propagation and its generalizations," Mitsubishi Elect. Res., vol. Tech. Rep. TR-2001-22, 2001.

[24] Y. Boykov, O. Veksler, and R. Zabin, "Fast approximate energy minimization via graph cuts," IEEE Pattern Anal. Mach. Intell., vol. 23, no. 11, pp. 1222-1239, Nov. 2001.

[25] V. Kolmogorov and R. Zabih, "What energy functions can be minimized via graph cuts?," IEEE Pattern Anal. Mach. Intell., vol. 26, no. 2, pp. 147-159, Feb. 2004.

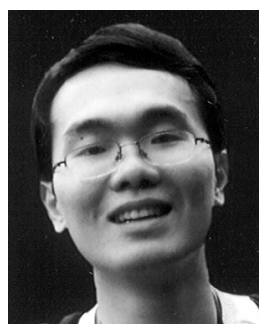

Jue $\mathbf{W u}\left(\mathrm{S}^{\prime} 04\right)$ received the B.Sc. degree in computer science from Fudan University, Shanghai, China, in 2003. He is currently pursuing the Ph.D. degree in bioengineering at The Hong Kong University of Science and Technology.

His research interests include biomedical image segmentation and registration, energy minimization, and statistical analysis.

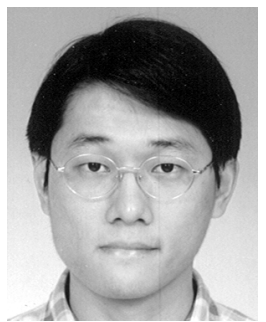

Albert C. S. Chung received the B.Eng. degree (first class Honors) in computer engineering from The University of Hong Kong in 1995 and the M.Phil. degree in computer science from The Hong Kong University of Science and Technology in 1998.

He joined the Medical Vision Laboratory, University of Oxford, Oxford, U.K., as a doctoral research student with a Croucher Foundation scholarship and graduated in 2001. He was a Visiting Scientist at the Artificial Intelligence Laboratory, Massachusetts Institute of Technology, Cambridge, in 2001. He is currently an Assistant Professor with the Department of Computer Science and Engineering, The Hong Kong University of Science and Technology. His research interests include medical image analysis, image processing, and computer vision.

Dr. Chung won the 2002 British Machine Vision Association Sullivan Thesis Award for the best doctoral thesis submitted to a U.K. university in the field of computer or natural vision. 